PROCEEDINGS OF THE

AMERICAN MATHEMATICAL SOCIETY

Volume 138, Number 3, March 2010, Pages 861-869

S 0002-9939(09)10168-5

Article electronically published on November 2, 2009

\title{
TWO CLASSES OF ALGEBRAS WITH INFINITE HOCHSCHILD HOMOLOGY
}

\author{
ANDREA SOLOTAR AND MICHELINE VIGUÉ-POIRRIER
}

(Communicated by Martin Lorenz)

\begin{abstract}
We prove without any assumption on the ground field that higher Hochschild homology groups do not vanish for two large classes of algebras whose global dimension is not finite.
\end{abstract}

\section{INTRODUCTION}

Let $k$ be a fixed field. All the algebras we consider are associative unital $k$ algebras. We will denote $\otimes=\otimes_{k}$.

It is well known that the homological properties of an algebra are related to the properties of its Hochschild (co)homology groups. For example, if a finite dimensional algebra over an algebraically closed field has finite global dimension, then all its higher Hochschild cohomology groups vanish. In [12, D. Happel said that he did not know whether the converse was true or not. It has been shown in [5] that it does not hold for algebras of type $A_{q}=k\langle x, y\rangle /\left(x^{2}, y^{2}, x y-q y x\right)$, where $q \in k$.

In 11, Han proved that the total Hochschild homology of the algebras $A_{q}$ is infinite dimensional. This fact led him to suggest the following conjecture:

Conjecture (Han). Let $A$ be a finite dimensional $k$-algebra. If the total Hochschild homology of $A$ is finite dimensional, then $A$ has finite global dimension.

In the same paper, Han provided a proof of this statement for monomial finite dimensional algebras.

Avramov and Vigué's computations in [1] show that Han's conjecture holds in the commutative case not only for finite dimensional algebras but for essentially finitely generated ones; see also 18 .

In 4, Han's conjecture is proved for graded local algebras, Koszul algebras and graded cellular algebras, provided the characteristic of the ground field is zero. The proof relies on the properties of the graded Cartan matrix and the logarithm and strongly uses the hypothesis on the characteristic of the field.

Received by the editors June 12, 2009, and, in revised form, August 16, 2009

2010 Mathematics Subject Classification. Primary 16E40, 16W50.

Key words and phrases. Global dimension, Hochschild homology theory.

This work was supported by the projects UBACYTX212 and PIP-CONICET 5099. The first author is a research member of CONICET (Argentina) and a Regular Associate of ICTP Associate Scheme.

The second author is a research member of University of Paris 13, CNRS, UMR 7539 (LAGA). 
In 3], the authors compute the Hochschild homology groups of quantum complete intersections; that is, algebras of type $A=k\langle x, y\rangle /\left(x^{a}, y^{b}, x y-q y x\right)$, where $q \in k^{*}$ is not a root of unity and $a, b \geq 2$ are fixed integers. In particular they prove Han's conjecture for this class of finite dimensional algebras.

The main purpose of this paper is to prove that higher Hochschild homology groups do not vanish for two large classes of algebras whose global dimension is not finite, without any assumption on the ground field.

In Theorem I, the algebras we consider are generalizations of quantum complete intersections, and they are not assumed to be finite dimensional.

On the other hand, the algebras satisfying the hypotheses of Theorem II are, in some sense, opposite of quantum complete intersections, since we assume that they have two generators $x$ and $y$ such that $x y=y x=0$.

Now we state both main theorems.

Theorem I. Let $A=k\left\langle x_{1}, \ldots, x_{n}\right\rangle /\left(f_{1}, \ldots, f_{p}\right)$ be a finitely generated $k$-algebra, such that $f_{1}$ belongs to $k\left[x_{1}\right]$ and, for $i \geq 2, f_{i}$ belongs to the two-sided ideal $\left(x_{2}, \ldots, x_{n}\right)$. If $B=k\left[x_{1}\right] /\left(f_{1}\right)$ is not smooth, then the Hochschild homology groups $H H_{n}(A)$ are not zero for all $n \in \mathbb{N}$.

For example Theorem I is valid if $f_{1}=x_{1}^{2} g_{1}$, with $g_{1} \in k\left[x_{1}\right]$ and $f_{2}, \ldots, f_{p}$ satisfying the hypothesis of the theorem.

Theorem II. Let $A=\bigoplus_{n \geq 0} A^{n}$ be a finite dimensional graded $k$-algebra with $A^{0}=$ $k$ and such that $\bar{A}=\bigoplus_{n>1} A^{n}$ is not zero. Assume that there exist two generators $x$ and $y$ of the algebra $A$ verifying $x y=y x=0$. Then the total Hochschild homology of $A$ is not finite dimensional.

Remark 1.1. This theorem is valid for very large classes of graded local algebras since relations between the other generators play no role.

The proof of Theorem I follows without any computation from the well known result for commutative algebras.

The methods used in the proof of Theorem II rely on differential homological algebra. In fact, we will work with the cobar construction on the graded coalgebra $\bigoplus_{n \geq 0} \operatorname{Hom}_{k}\left(A^{n}, k\right)$. We denote it $\left(\Omega^{*} A, d\right)$. The Hochschild homology groups of the differential graded algebra $\left(\Omega^{*} A, d\right)$ are dual, as vector spaces, to the Hochschild homology groups of the graded $k$-algebra $A$. Since $\left(\Omega^{*} A, d\right)$ is a tensor algebra, a short complex is available to compute its Hochschild homology.

The paper is organized as follows:

(1) Introduction

(2) Proof of Theorem I

(3) Interpretation in terms of quivers

(4) Hochschild homology in the differential graded case

(5) Proof of Theorem II

\section{Proof of Theorem I}

Let $A$ be an associative unital $k$-algebra. The definition of the Hochschild homology groups, $H H_{n}(A), n \geq 0$, is well known (see for example [13]). We have

$$
H H_{n}(A):=\operatorname{Tor}_{n}^{A^{e}}(A, A)=H_{n}\left(C_{*}(A), b\right),
$$


where $\left(C_{*}(A), b\right)$ is the Hochschild complex of $A$. Clearly, $H H_{n}(A)$ is a $k$-vector space for all $n \geq 0$.

In this section we assume that $A=k\left\langle x_{1}, \ldots, x_{n}\right\rangle /\left(f_{1}, \ldots, f_{p}\right)$, where $n, p \geq 1$; that $f_{1}$, which we may suppose is monic, belongs to $k\left[x_{1}\right]$; and that, for $i \geq 2$, $f_{i}$ belongs to the two-sided ideal $\left(x_{2}, \ldots, x_{n}\right)$. Let us consider the $k$-algebra $B=$ $k\left[x_{1}\right] /\left(f_{1}\right)$ and the maps

$$
\begin{gathered}
\iota: B \rightarrow A \text { with } \iota\left(x_{1}\right)=x_{1}, \\
\pi: A \rightarrow B \text { with } \pi\left(x_{1}\right)=x_{1}, \pi\left(x_{i}\right)=0, \text { for } i \geq 2 .
\end{gathered}
$$

The following lemma is easy to prove.

Lemma 2.1. The maps $\iota$ and $\pi$ are morphisms of $k$-algebras and satisfy $\pi \circ \iota=i d_{B}$.

Now, Theorem I is an immediate consequence of the following facts:

- the morphisms $\iota$ and $\pi$ induce by functoriality $k$-linear maps

$H H_{*}(\iota): H H_{*}(B) \rightarrow H H_{*}(A)$ and $H H_{*}(\pi): H H_{*}(A) \rightarrow H H_{*}(B)$

satisfying $H H_{*}(\pi) \circ H H_{*}(\iota)=i d_{H H_{*}(B)}$

- using a result of [1] $H H_{n}(B)$ is nonzero for an infinite sequence of integers $n$.

Another proof can be given using the computations for $H H_{n}(B)$ in [6]: if $f_{1}$ and $f_{1}^{\prime}$ are not coprime, then $H H_{n}(B) \neq 0$ for all $n \in \mathbb{N}$.

Example 2.2. If $f_{1}=x_{1}^{a}$, with $a \geq 2$, and $f_{i} \in\left(x_{2}, \ldots, x_{n}\right)$, then Theorem I holds. This covers the case of quantum complete intersections.

An interesting question is to ask if the algebras $A$ considered in Theorem I have infinite global dimension. In the commutative case, it is well known that this is true. Also, if $A=k\left\langle x_{1}, \ldots, x_{n}\right\rangle /\left(f_{1}, \ldots, f_{p}\right)$ is a finite dimensional $k$-vector space, Happel's result 12 implies that $\operatorname{gldim}(A)=\infty$, where gldim denotes the global dimension of the algebra.

It follows from Serre's theorem on page 37 of 15$]$ that if $B$ is not smooth, then its global dimension is not finite. In the general case, we cannot ensure that if we have $k$-algebras $A$ and $B$ as above with $\operatorname{gldim}(B)=\infty$, then $\operatorname{gldim}(A)=\infty$.

However, we can use the algebra map $\iota: B \rightarrow A$ to obtain that the global dimension of $A$ is not finite in some cases: Suppose that $\iota$ endows $A$ with a structure of a flat $B$-module. In this situation, Corollary 4.4 of [2] says that $\operatorname{gldim}(A)=\infty$. This is the case, for example, of quantum complete intersections.

\section{INTERPRETATION IN TERMS OF QUIVERS}

Let $A$ be a $k$-algebra which is isomorphic to $k Q^{A} / I^{A}$ for a given finite quiver $Q^{A}$ and an admissible ideal $I^{A}$. In case $k$ is an algebraically closed field and $A$ is finite dimensional and basic, there always exist a quiver and an admissible ideal such that the above isomorphism holds.

Let us denote by $Q_{0}^{A}=\left\{e_{1}, \ldots, e_{r}\right\}$ the set of vertices of $Q^{A}$ and by $Q_{1}^{A}$ its set of arrows. Then $k Q_{0}^{A}$ is an algebra, $k Q_{1}^{A}$ is a $k Q_{0}^{A}$ two-sided ideal and $A=$ $T_{k Q_{0}^{A}} k Q_{1}^{A} / I^{A}$, where $I^{A} \subseteq\left(k Q_{1}^{A}\right)^{2}$.

Suppose that there exist $e_{i} \in k Q_{0}^{A}$ and $x \in e_{i}\left(k Q_{1}^{A}\right) e_{i}$. In fact, since $A$ is finite dimensional and $I^{A}$ is admissible, if such a loop $x$ exists, then $x^{n}=0$ for some integer $n \geq 2$. 
Let $B$ be the $k$-algebra $k[x] /\left\langle x^{n}\right\rangle$. Then $B=T_{k Q_{0}^{B}} k Q_{1}^{B} / I^{B}$, where $Q_{0}^{B}=\left\{e_{i}\right\}$, $Q_{1}^{B}=\{x\}$ and $I^{B}=\left\langle x^{n}\right\rangle$.

We may consider the morphisms of algebras of the previous section. In this case the map $\iota$ is completely determined by its values on $e_{i}$ and $x$. It sends $e_{i}$ to $e_{1}+\cdots+e_{r}$ and $x$ to $x$. Clearly, it is well defined.

On the other hand, the morphism $\pi: A \rightarrow B$ is given as follows: $\pi\left(e_{j}\right)=\delta_{i j} e_{i}$, for $1 \leq j \leq r$, and the restriction of $\pi$ to the arrows of $A$ is given by $\pi(y)=$ $\delta_{y x} x$, where $\delta$ is the Kronecker delta. If we assume that $I^{A}=\left\langle x^{n}, f_{2}, \ldots, f_{s}\right\rangle$ is admissible and that $f_{i}$ belongs to the two-sided ideal generated by $Q_{1}^{A}-\{x\}$, then it is straightforward to check that $\pi$ is also well defined and $\pi \circ \iota=i d_{B}$.

As a consequence of the results of Section 2, we see that the Hochschild homology dimension, denoted $h \operatorname{dim}(B)$, is infinite, and so the same holds for $A$. Being both $k$-finite dimensional, their global dimensions cannot be finite.

It is interesting to note that whenever $A$ and $B$ are finite dimensional $k$-algebras provided by morphisms $\pi$ and $\iota$ satisfying $\pi \circ \iota=i d$ as in the previous section and $B$ has infinite Hochschild dimension, $\operatorname{hhdim}(A)$ will also be infinite. For example, let $\operatorname{char}(k)=0$ and $B$ be a finite dimensional monomial $k$-algebra with generators $x_{1}, \ldots, x_{r}$ such that $\operatorname{hhdim}(B) \neq 0$, and let $A$ be a finite dimensional $k$-algebra generated by $x_{1}, \ldots, x_{r}, \ldots, x_{n}$ such that $B \cong A /\left\langle f_{1}, \ldots, \mathfrak{f}_{s}\right\rangle$ and $f_{i}$ belongs to the two-sided ideal $\left\langle x_{r+1}, \ldots, x_{n}\right\rangle, 1 \leq i \leq s$. Then $\operatorname{hhdim}(B)=\infty$ [11, and the same is true for $A$.

\section{Hochschild homology in the Differential gRAded CASE}

In this section we deal with finite dimensional algebras.

4.1. Notation. We use the methods of differential graded algebra of [7]. In particular an element of lower degree $i \in \mathbb{Z}$ is, by the classical convention, of upper degree $-i$. All the algebras considered from now on are unital, associative, with a differential of degree -1 . We recall that if $V=\bigoplus_{i \in \mathbb{Z}} V_{i}$ is a graded $k$-vector space, then the suspended graded $k$-vector space $s V$ has homogeneous components $(s V)_{i}=V_{i-1}$, for $i \in \mathbb{Z}$. The $k$-algebra $T V$ will denote the tensor algebra on $V$. The degree of an element $v \in V$ is denoted by $|v|$.

For any differential graded algebra $A$, let $A^{o p}$ be the opposite graded algebra, and $A^{e}=A \otimes A^{o p}$ be the enveloping algebra. The categories of graded $A$-bimodules and of left (or right) differential graded $A^{e}$-modules are equivalent.

4.2. Bar resolution and Hochschild homology. Let $(A, d)$ be an augmented algebra and let $\bar{A}=\operatorname{Ker}(\epsilon: A \rightarrow k)$. The normalized bar resolution of $A$, denoted by $B(A, A, A)$, is the differential graded $A^{e}$-module $\left(A \otimes T(s \bar{A}) \otimes A, D_{0}+D_{1}\right)$, where $D_{0}$ is the differential induced by $d$ on the tensor product of complexes and $D_{1}$ is defined as follows (see for example [9], 2.2):

$$
\begin{aligned}
D_{1}\left(a \otimes s a_{1} \otimes \cdots \otimes s a_{n} \otimes b\right)= & (-1)^{|a|} a a_{1} \otimes s a_{2} \otimes \cdots \otimes s a_{n} \otimes b \\
& \pm \sum_{i=1}^{n-1} a \otimes s a_{1} \otimes \cdots \otimes s\left(a_{i} a_{i+1}\right) \otimes \cdots \otimes s a_{n} \otimes b \\
& \pm a \otimes s a_{1} \otimes \cdots \otimes s a_{n-1} \otimes a_{n} b .
\end{aligned}
$$

The Hochschild homology of the differential graded algebra $(A, d)$ is, by definition, the graded vector space $\mathcal{H} \mathcal{H}_{*}(A)=\operatorname{Tor}_{*}^{A^{e}}(A, A)$ in the differential sense of [14]. 
Lemma 4.1 ([7]). The canonical map $m: B(A, A, A) \rightarrow A$ defined by 0 on $A \otimes$ $T^{\geq 1}(s \bar{A}) \otimes A$ and by multiplication on $A \otimes A$ is a semifree resolution of $A$ as an $A^{e}$-module.

Consequently we have,

$$
\mathcal{H H}_{*}(A, d)=H_{*}\left(\mathcal{C}_{*}(A), \delta\right)
$$

with

$$
\mathcal{C}_{*}(A)=A \otimes_{A^{e}} B(A, A, A)=A \otimes T(s \bar{A}),
$$

and $\delta=\delta_{0}+\delta_{1}$, where $\delta_{0}$ and $\delta_{1}$ are obtained by tensorization. Explicitly,

$$
\begin{aligned}
\delta_{1}\left(a \otimes s a_{1} \otimes \cdots \otimes s a_{n}\right)= & (-1)^{|a|} a a_{1} \otimes s a_{2} \otimes \cdots \otimes s a_{n} \\
& +\sum_{i=1}^{n-1}(-1)^{\epsilon_{i}} a \otimes s a_{1} \otimes \cdots \otimes s\left(a_{i} a_{i+1}\right) \otimes \cdots \otimes s a_{n} \\
& +(-1)^{\epsilon_{n}} a_{n} a \otimes s a_{1} \otimes \cdots \otimes s a_{n-1},
\end{aligned}
$$

where the $\epsilon_{i}$ 's are integers depending on the degrees of the elements $a_{i}$. If all these degrees are even, then $\epsilon_{i}=i$.

In the rest of this paper we consider only differential graded algebras $(A, d)$ satisfying either condition (a) or condition (b) below:

(a) $A_{n}=0$ for $n<0$ and $A_{0}=k$, so that $\mathcal{C}_{n}(A)=0$ for $n<0$;

(b) $A_{n}=0$ for $n>0, A_{0}=k$, and $A_{-1}=0$, so that $\mathcal{C}_{n}(A)=0$ for $n>0$.

In both cases, we have $\mathcal{C}_{0}(A)=k$.

4.3. Cobar construction and duality construction in Hochschild homology. We next recall the definition of the cobar construction described in Section 19 of [8]. Let $\left(C, d_{C}\right)$ be a coaugmented differential graded coalgebra with comultiplication $\Delta$, and let $\bar{C}=\operatorname{Ker}(\epsilon: C \rightarrow k)$. We denote $(\Omega C, d)$ as the augmented differential graded algebra defined as follows:

- $\Omega C=T\left(s^{-1} \bar{C}\right)$, as an augmented graded algebra;

- $d=d_{0}+d_{1}$, with $d_{0}\left(s^{-1} c\right)=-s^{-1}\left(d_{C}(c)\right)$, if $c \in \bar{C}$, and $d_{1}$ is defined from $\Delta$.

Suppose now that $\left(A, d_{A}\right)$ is a finite dimensional differential graded algebra. Then the graded dual $A^{\vee}=H_{A} m_{k}(A, k)$ is a differential graded coalgebra with differential $d_{A}^{\vee}$, the transpose of $d_{A}$.

Definition 4.2. $\left(\Omega^{*} A, d\right):=\left(\Omega\left(A^{\vee}\right), d\right)$, where $d$ is defined from $d_{A}^{\vee}$ and the comultiplication of $A^{\vee}$ as above.

We have $\Omega^{*} A=T(V)$ with $V=H_{o m}(s \bar{A}, k)$. If $\left(A, d_{A}\right)$ satisfies condition (b) above, then

$$
V=\bigoplus_{n \geq 1} V_{n}, \text { with } V_{n}=\operatorname{Hom}_{k}\left(A_{-n-1}, k\right)
$$

and then $\left(\Omega^{*} A, d\right)$ satisfies condition (a). Similarly, if $\left(A, d_{A}\right)$ satisfies condition (a), then $\left(\Omega^{*} A, d\right)$ satisfies condition (b).

The first ingredient used to prove Theorem II is the following duality property.

Theorem 4.3 (10, [16]). Let $\left(A, d_{A}\right)$ be a finite dimensional algebra satisfying either condition (a) or (b) above. Then for all $n \in \mathbb{Z}$ we have

$$
\operatorname{Hom}_{k}\left(\mathcal{H} \mathcal{H}_{-n}(A), k\right)=\mathcal{H H}_{n}\left(\Omega^{*} A\right) \text {. }
$$


Consequently, the computation of the graded vector space $\mathcal{H H}_{n}(A)$ can be replaced by the computation of the Hochschild homology of a quasifree differential graded algebra $(T(V), d)$.

4.4. A short complex for the computation of the Hochschild homology. Now, we want to compute the Hochschild homology of $(T(V), d)$, with $V=$ $\bigoplus_{n \geq 1} V_{n}$.

We recall here the main results of [17]. Put $(T(V), d)=(B, d)$ and let $P=$ $(B \otimes B) \oplus(B \otimes(s V) \otimes B)$. We define a differential $D$ on $P$, which is the tensor product of the differentials on $B \otimes B$, and

$$
D(a \otimes s v \otimes b)=d a \otimes s v \otimes b \pm(a v \otimes b-a \otimes v b)+S(a \otimes s v \otimes b),
$$

where $S(a \otimes s v \otimes b) \in B \otimes s V \otimes B$, for $a \in B, b \in B$ and $v \in V$.

Proposition 4.4 (Thm. 1.4 in [17]). The canonical map $m:(P, D) \rightarrow B$ defined as 0 on $B \otimes s V \otimes B$ and as multiplication on $B \otimes B$ is a semifree resolution of $B$ as a $B^{e}$-module.

As a consequence,

$$
\mathcal{H H}_{*}(T(V), d)=H_{*}\left(B \otimes_{B^{e}} P, \delta\right),
$$

with differential $\delta=d \otimes_{B^{e}} D$ that will be made precise in the next section. We have:

- $\delta_{\mid T(V)}=d$

- $\delta(a \otimes s v)=d a \otimes s v+(-1)^{|a|}\left(a v-(-1)^{|v| \times|a|} v a\right)-\sigma(a \otimes d v)$, where $\sigma(a \otimes d v)$ belongs to $T(V) \otimes s V$, for $a \in T(V), v \in V$.

Put $Q_{*}:=B \otimes_{B^{e}} P=T(V) \oplus(T(V) \otimes s V)$.

Theorem 4.5 (Thm. 1.5 of [17]). With the above notation,

$$
\mathcal{H H}_{*}(T(V), d)=H_{*}\left(Q_{*}, \delta\right) \text {. }
$$

In the following section we will use the complex $\left(Q_{*}, \delta\right)$ to compute the Hochschild homology of a finite dimensional graded algebra $A=\bigoplus_{n \geq 0} A^{n}$, with $A^{0}=k$. In this case, the graded vector space $V$ is also finite dimensional, and the differential $\delta$ has good properties.

\section{Proof of Theorem II}

We work with a finite dimensional graded algebra with $A^{0}=k$. We may assume without loss of generality that $A$ is graded in even degrees, $A=k \oplus\left(\bigoplus_{n \geq 2} A^{n}\right)$, and $\bar{A}=\bigoplus_{n \geq 2} A^{n}$ is nonzero.

5.1. Relations between $H H_{*}(A)$ and $\mathcal{H H}_{*}(A, 0)$. Using the conventions recalled at the beginning of the previous section, we consider $A$ as a differential graded algebra with differential 0 and $A_{-n}=A^{n}$.

Since $A$ is graded, the ordinary Hochschild homology $H H_{*}(A)$ defined in Section 2 is graded, and there is a decomposition

$$
H H_{*}(A)=\bigoplus_{p, q \geq 0} H H_{p}(A)^{q} .
$$

Since $A$ is finite dimensional, $H H_{p}(A)$ is finite dimensional for all $p$. 
Lemma 5.1. Let $A$ be an algebra as above. Then,

(1) $\mathcal{H H}_{*}(A, 0)=\bigoplus_{n>0} \mathcal{H} \mathcal{H}_{-n}(A)$ and $\mathcal{H H}_{-n}(A)=\bigoplus_{p} H H_{p}(A)^{p+n}$.

(2) $H H_{p}(A)^{p+n}=0$ if $p>n$ or $p<\frac{n-N}{N-1}$, where $N=\sup \left\{n \mid A^{n} \neq 0\right\}$.

Corollary 5.2. If there exists an increasing sequence of integers $n_{i}$ such that $\mathcal{H H}_{-n_{i}}(A) \neq 0$, then $H_{*}(A)$ is not finite dimensional.

The strategy now is to focus our attention on $\mathcal{H H}_{*}\left(\Omega^{*} A\right)$ using Theorem 4.3 , But Theorem 4.5 allows us to use the short complex $\left(Q_{*}, \delta\right)$ to compute $\mathcal{H H}_{*}\left(\Omega^{*} A\right)$, so we will work with this last one.

5.2. Description of $\left(Q_{*}, \delta\right)$. Let $A=k \oplus\left(\bigoplus_{n \geq 2} A^{n}\right)$ be a finite dimensional graded algebra. We fix a homogeneous linear basis $\left(a_{i}\right)_{i \in I}$ for $\bar{A}=\bigoplus_{n>2} A^{n}$. This choice determines the structure constants $\alpha_{j k}^{i}$ by the equalities $a_{j} a_{k}=\sum \alpha_{j k}^{i} a_{i}$.

In this situation, $(\bar{A})^{\vee}=\operatorname{Hom}_{k}(\bar{A}, k)$ is endowed with the dual basis $\left(b_{i}\right)_{i \in I}$ satisfying $\left\langle b_{i}, a_{j}\right\rangle=\delta_{i j}$. Notice that $A^{\vee}$ is a graded coalgebra with comultiplication $\Delta$, and $\Delta b_{i}=\sum_{j . k} \beta_{i}^{j k} b_{j} \otimes b_{k}$, where $\alpha_{j k}^{i}=(-1)^{\left|a_{j}\right| \times\left|a_{k}\right|} \beta_{i}^{j k}$.

We have already defined $\left(\Omega^{*} A, d\right)=\left(\Omega\left(A^{\vee}\right), d\right)=(T(V), d)$. Now, put $v_{i}=$ $s^{-1} b_{i}$; then $\left|v_{i}\right|=n-1$ if $a_{i} \in A^{n}$. We check that

$$
d v_{i}=\sum_{j, k}(-1)^{\left|a_{j}\right|+\left|a_{j}\right| \times\left|a_{k}\right|} \alpha_{j k}^{i} v_{j} \otimes v_{k} .
$$

So $\left(\Omega^{*} A, d\right)=(T(V), d)$ is a tensor algebra with a quadratic differential.

Furthermore, we have assumed without loss of generality that $A$ is graded in even degrees, so that $V$ is graded only in odd degrees. In this case, we give an explicit formula for the differential $\delta$ on $Q_{*}$ (cf. Subsection 4.4).

Put $\bar{V}=s V$; then $Q_{*}=T(V) \oplus T(V) \otimes \bar{V}$. Let $v$ be an element in $V$, and let $d v=\sum_{j, k} \lambda_{j k} v_{j} \otimes v_{k}$ with $\lambda_{j k} \in k$. Let $a$ be an element in $T(V)$.

We have

$$
\delta(a \otimes \bar{v})=d a \otimes \bar{v}+(-1)^{|a|}\left(a v-(-1)^{|a|} v a\right)-\sigma(a \otimes d v),
$$

where

$$
\sigma(a \otimes d v)=-(-1)^{|a|} \sum_{j, k} \lambda_{j k} a v_{j} \otimes \bar{v}_{k}+\sum_{j, k} \lambda_{j k} v_{k} a \otimes \bar{v}_{j} .
$$

5.3. A nice homogeneous basis $\left(a_{i}\right)$ for $\bar{A}$. Since $A=k \oplus \bar{A}$, the projection $\bar{A} \rightarrow \bar{A} / \bar{A}^{2}=U$ has a section $\rho$ that extends to a morphism of algebras $T(U) \rightarrow A$ whose kernel is contained in $T^{\geq 2}(U)$. This implies that $\left(x_{i}\right)_{1 \leq i \leq p}$ are generators of the algebra $A$ if and only if their images in $\bar{A} / \bar{A}^{2}$ form a basis of this vector space.

As vector spaces, $\bar{A}=\bar{A} / \bar{A}^{2} \oplus \bar{A}^{2}$, and we will consider a homogeneous basis of $\bar{A} / \bar{A}^{2}$ and a basis of $\bar{A}^{2}$. If $a_{i} \in \bar{A} / \bar{A}^{2}$, then the corresponding $v_{i}$ in $\left(\Omega^{*} A, d\right)$ satisfies $d v_{i}=0$.

We will now prove the following result.

Theorem 5.3. Let $A=\bigoplus_{n>0} A^{n}$ be a finite dimensional graded $k$-algebra with $A^{0}=k$, such that $\bar{A}=\bigoplus_{n>1} \bar{A}^{n}$ is not zero. Assume that there exist two generators $x$ and $y$ of the algebra $A$ satisfying $x y=y x=0$. Then $H_{n_{i}}\left(Q_{*}, \delta\right) \neq 0$ for a strictly increasing sequence of integers $\left(n_{i}\right)$. 
Proof. We can associate to $x$ and $y$ two elements $a_{1}$ and $a_{2}$, linearly independent in $\bar{A}$. We denote by $v_{1}$ and $v_{2}$ the corresponding elements in a dual basis of $V$. If $\left(a_{1}, \ldots, a_{n}\right)$ is a linear basis of $\bar{A}$ and $\left(v_{1}, \ldots, v_{n}\right)$ is the corresponding basis of $V$, then we have $d v_{1}=0, d v_{2}=0$ and, for $i \geq 3$,

$$
d v_{i}=\sum_{j, k} \alpha_{j k}^{i} v_{j} \otimes v_{k}
$$

The fact that $x y=y x=0$ implies that, for $i \geq 3, \alpha_{12}^{i}=\alpha_{21}^{i}=0$.

For $n \geq 1$, consider

$X_{n}=v_{1} \otimes v_{2} \otimes v_{1} \otimes v_{2} \otimes \cdots \otimes v_{1} \otimes \bar{v}_{2}-v_{2} \otimes v_{1} \otimes v_{2} \otimes v_{1} \otimes \cdots \otimes v_{2} \otimes \bar{v}_{1} \in V^{\otimes(2 n-1)} \otimes \bar{V}$.

It is easy to see that $\left|X_{n}\right|=n\left(\left|v_{1}\right|+\left|v_{2}\right|\right)+1$ and that $\delta X_{n}=0$.

If $X_{n}$ was a boundary, there should exist $Y, b_{i} \in T(V)$ such that

$$
X_{n}=\delta\left(Y+\sum_{i} b_{i} \otimes \bar{v}_{i}\right)
$$

and

$$
X_{n}=d Y+\sum_{i} d b_{i} \otimes \bar{v}_{i}+\sum_{i}\left(b_{i} v_{i}-v_{i} b_{i}\right)+\sum_{i} \alpha_{j k}^{i} b_{i} v_{j} \otimes \bar{v}_{k}-\sum_{i} \alpha_{j k}^{i} v_{k} b_{i} \otimes \bar{v}_{j} .
$$

Such elements cannot exist since, for all $i$,

$$
d v_{i}=\sum_{j, k} \alpha_{j k}^{i} v_{j} \otimes v_{k} \text { with } \alpha_{12}^{i}=\alpha_{21}^{i}=0 .
$$

Example 5.4. Let $A=k\langle x, y, z\rangle /\left(x y, y x, x^{2}-y^{2}, x^{2}-z^{2}, x z-q z x, y z-q z y\right)$, where $q \in k, q^{2} \neq 1$ and -1 is not a square in $k$. This example is not covered by Theorem I.

\section{REFERENCES}

1. Avramov, L.; Vigué-Poirrier, M. Hochschild homology criteria for smoothness. Internat. Math. Res. Notices 1 (1992), 17-25. MR1149001 (92m:13020)

2. Bavula, V. V. Tensor homological minimal algebras, global dimension of the tensor product of algebras and of generalized Weyl algebras, Bull. Sci. Math. 120 (1996), no. 3, 293-335. MR.1399845 (97f:16015)

3. Bergh, P. A.; Erdmann, K. Homology and cohomology of quantum complete intersections. Algebra Number Theory 2 (2008), no. 5, 501-522. MR2429451 (2009h:16010)

4. Bergh, P. A.; Madsen, D. Hochschild homology and global dimension. Bull. London Math. Soc. 41 (2009), no. 3, 473-482. MR2506831

5. Buchweitz, R.; Green, E.; Madsen, D.; Solberg, Ø. Finite Hochschild cohomology without finite global dimension. Math. Res. Lett. 12 (2005), 805-816. MR2189240 (2006k:16019)

6. Buenos Aires Cyclic Homology Group. Cyclic homology of algebras with one generator. (J. A. Guccione, J. J. Guccione, M. J. Redondo, A. Solotar and O. Villamayor participated in this research.) $K$-Theory 5 (1991), 51-69. MR.1141335 (93b:19002)

7. Félix, Y.; Halperin, S.; Thomas, J.-C. Differential graded algebras in topology. Handbook of algebraic topology, 829-865, North-Holland, Amsterdam, 1995. MR1361901 (96j:57052)

8. Félix, Y.; Halperin, S.; Thomas, J.-C. Rational homotopy theory. Graduate Texts in Mathematics, vol. 205, Springer-Verlag, New York, 2001. MR1802847 (2002d:55014)

9. Félix, Y.; Thomas, J.-C.; Vigué-Poirrier, M. The Hochschild cohomology of a closed manifold. Publ. Math. Inst. Hautes Études Sci. 99 (2004), 235-252. MR.2075886 (2005g:57054)

10. Halperin, S.; Vigué-Poirrier, M. The homology of a free loop space. Pacific J. Math. 147 (1991), no. 2, 311-324. MR1084712 (92e:55012)

11. Han, Y. Hochschild (co)homology dimension. J. London Math. Soc. (2) 73 (2006), no. 3, 657-668. MR2241972 (2007c:16018) 
12. Happel, D. Hochschild cohomology of finite-dimensional algebras. Séminaire d'Algèbre Paul Dubreil et Marie-Paul Malliavin, 39ème Année (Paris, 1987/1988), 108-126, Lecture Notes in Math., 1404, Springer, Berlin, 1989. MR1035222 (91b:16012)

13. Loday, J.-L. Cyclic homology. Appendix E by M. Ronco. Second edition. Chapter 13 by the author in collaboration with Teimuraz Pirashvili. Grundlehren der Mathematischen Wissenschaften, vol. 301, Springer-Verlag, Berlin, 1998. MR.1600246 (98h:16014)

14. MacLane, S. Homology. Reprint of the first edition. Die Grundlehren der Mathematischen Wissenschaften, vol. 114, Springer-Verlag, Berlin-New York, 1967. MR0349792 (50:2285)

15. Serre, J.-P. Algèbre locale. Multiplicités. Lecture Notes in Math., 11, Springer-Verlag, Berlin, 1965. MR0201468 (34:1352)

16. Solotar, A. Cyclic homology of a free loop space. Comm. Algebra 21 (1993), no. 2, 575-582. MR.1199690 (94h:19003)

17. Vigué-Poirrier, M. Homologie de Hochschild et homologie cyclique des algèbres différentielles graduées. International Conference on Homotopy Theory (Marseille-Luminy, 1988). Astérisque, vol. 191, Soc. Math. France, 1990, pp. 255-267. MR.1098974 (92e:19003)

18. Vigué-Poirrier, M. Critères de nullité pour l'homologie des algèbres graduées. C. R. Acad. Sci. Paris Sér. I Math. 317 (1993), no. 7, 647-649. MR1245091 (94g:19003)

Departamento de Matemática, Facultad de Ciencias Exactas y Naturales, Universidad de Buenos Aires, Ciudad Universitaria, Pabellón 1 1428, Buenos Aires, Argentina

E-mail address: asolotar@dm.uba.ar

Laboratoire Analyse, Géométrie et Applications, UMR CNRS 7539, Institut Galilée, Université Paris 13, F-93430 Villetaneuse, France

E-mail address: vigue@math.univ-paris13.fr 